\title{
Identification and characterization of Colletotrichum species causing grape ripe rot in southern China
}

\author{
Lei $\mathrm{Y}^{1,2}$, Tang $\mathrm{XB}^{1,2}$, Jayawardena $\mathrm{RS}^{3,4}$, Yan $\mathrm{JY}^{3}$, Wang $\mathrm{XD}^{2}$, Liu $\mathrm{M}^{3}$, Chen $\mathrm{T}^{2}$, \\ Liu $\mathrm{XM}^{2}$, Wang $\mathrm{JC}^{2}$, Chen $\mathrm{QX}^{{ }^{*}}$
}

${ }^{1}$ College of Horticulture, Fujian Agriculture and Forestry University, Fuzhou 350002, Fujian, China

${ }^{2}$ Fruit Research Institute, Fujian Academy of Agriculture Science, Fuzhou 350013, Fujian, China

${ }^{3}$ Institute of Plant and Environmental Protection, Beijing Academy of Agriculture and Forestry Sciences, Beijing, 100097, China

${ }^{4}$ Center of Excellence in Fungal Research, School of Science, Mae Fah Luang University, Chiang Rai 57100, Thailand

Lei Y, Tang XB, Jayawardena RS, Yan JY, Wang XD, Liu M, Chen T, Liu XM, Wang JC, Chen QX 2016 - Identification and characterization of Colletotrichum species causing grape ripe rot in southern China. Mycosphere 7(8) 1177-1191, Doi 10.5943/mycosphere/si/2c/8

\begin{abstract}
Grape ripe rot is known to be causing serious losses in grapes in many countries. In order to clarify the species of pathogen causing grape ripe rot in southern China, 62 samples with ripe rot symptoms were collected from Fujian and Guizhou Provinces. Single spore isolation techniques resulted in 50 isolates of Colletotrichum species. Based on multigene data and morphology, 48 isolates were identified as $C$. viniferum and one isolate was identified as $C$. citri. A Colletotrichum species, sister to $C$. cliviae, was identified to be causing grape ripe rot. However, until further collections are made, this species will be remain as Colletotrichum sp. This is the first time that $C$. citri and the undetermined Colletotrichum species sister to $C$. cliviae have been reported to be associated with grape ripe rot in the world.
\end{abstract}

Key words - China - Colletotrichum citri - Grape ripe rot - Identification - Pathogenicity

\section{Introduction}

As one of the first fruits cultivated worldwide, grapes play a key role in winemaking as well as processed grape products, such as jam and raisins (Hong et al. 2008). Since 1990s, with the breakthrough in cultivation techniques and the changes in the structure of agricultural policy, the grape industry in Fujian Province has grown rapidly, evidenced by a rapid increase of cultivated acreage and production (Lei et al. 2011). By the end of 2014, the total area of grape production was $8,527 \mathrm{hm}^{2}$, resulting in with more than 153,300 t of grapes (Fujian Provincial Bureau of Statistics 2015). In Guizhou Province, grapes are cultivated in nine cities, especially in the central and southeastern regions. The main variety is 'Crystal' with a cultivation area of approximately 17,000 $\mathrm{hm}^{2}$, which accounts for $60 \%$ of the total area in Guizhou Province (Tang 2015). However, Fujian Province and Guizhou Province both have warm summer conditions because of the humid subtropical seasonal and windy climate, resulting in the occurrence of a variety of pests in mature grapes (Lu 2005). 
Colletotrichum is a phytopathogenic genus that is widespread in the world, causing disease in various crops and fruits, such as banana, citrus, grape, mango and strawberry. It often causes huge economic losses especially in tropical and subtropical regions (Phoulivong et al. 2010). Colletotrichum species can invade multiple parts of the plant (leaf, flower, stem and fruit), causing a variety of symptoms such as fruit rot, dry shoot, and withering. Fruits are often infected in the period of pre- and post-harvest seasons (Bailey \& Jeger 1992). Grape ripe rot is one of the most important diseases in the mature stage of grape development. It was first reported in 1891 in the United States (Southworth 1891). Colletotrichum gloeosporioides s.l and C. acutatum were previously reported as the disease pathogens resulting in ripe rot of grapes (Daykin \& Miholland 1984, Yamamoto et al. 1999, Melksham et al. 2002). Identification of Colletotrichum species based solely on morphology is prone to errors because there are no definite morphotaxonomic characters and these characters may suffer from inconsistency when subjected to different experimental methods and conditions (Cai et al. 2009, Hyde et al. 2009). In recent years, a large number of species of Colletotrichum have been identified using morphological characteristics with the combination of multi-gene analysis (Moriwaki \& Tsukiboshi 2009, Weir \& Johnston 2010, Wikee et al. 2011, Peng et al. 2013). Peng et al. (2013) satisfactorily classified 26 isolates of Colletotrichum from Guizhou and Yunnan provinces and also described a new species, C. viniferum. Yan et al. (2015) isolated and identified 34 isolates from six provinces and reported the grape ripe rot caused by $C$. aenigma for the first time from China. Therefore, the results of molecular approaches are more stable and reliable, and would also be helpful in understanding the evolutionary relationships of Colletotrichum species (Cai et al. 2009, Hyde et al. 2009).

To determine the species of grape ripe rot and their pathogenicity differences, we collected 62 samples from Fujian and Guizhou Provinces. Based on morphology and multi-gene phylogeny, the species were identified.

\section{Materials \& Methods}

\section{Sample collection and isolation of fungi}

Fungi were isolated from grapevine fruits samples with typical symptoms of ripe rot from Fujian Province and Guizhou Province in July 2015 (Table 1). Grapevine varieties used in this study included $V$. vinifera $c v$. Kyoho, V. Labruscana $c v$. Summer Black and others listed in Table 1. Pathogens were isolated via purifying a single spore according to "Plant Pathology Research Methods" (Fang 1998). The grape variety used for the pathogenicity test was V. vinifera cv. Kyoho and was available from local farmer's market in Beijing, China.

\section{Morphological characterization}

Morphological and cultural characterization was carried out according to the method of Cai et al. (2009). The diseased fruits were disinfected with $0.1 \%$ Sodium hypochlorite and $70 \%$ ethanol for 1 min. A tissue block of approximately $0.3 \mathrm{~mm} \times 0.3 \mathrm{~mm} \times 0.3 \mathrm{~mm}$ was cut off from the junction of the healthy part and the lesion part and was re-disinfected with $70 \%$ ethanol for $2-3 \mathrm{~min}$, and then cultivated at $25{ }^{\circ} \mathrm{C}$ on a potato dextrose agar (PDA) medium. After the colonies were visible clearly, a block was taken from the edge of the mycelium with a diameter of $5 \mathrm{~mm}$ punches, and then transferred to a new PDA plate for 7-10 d cultivation. Afterwards, purification was performed by a single spore separation method. Mycelial discs ( $5 \mathrm{~mm}$ diameter) were cut off from the edge of five-day old colonies then transferred to a new PDA plate and incubated in the dark at $28{ }^{\circ} \mathrm{C}$. Each strain was repeated in triplicate. Colony diameter was measured after 7 days, and the average of growth rate $(\mathrm{mm} / \mathrm{day})$ was calculated. The morphology of both colonies and conidia was observed, the size (length and width) of 10 pycnidia and 50 conidia was measured under a microscope (Nikon SMZ1500 and AXIO Imager. Z2) (Weir et al. 2012). 
Table 150 isolates of Colletotrichum from this study.

\begin{tabular}{|c|c|c|c|}
\hline $\begin{array}{l}\text { Isolate } \\
\text { no. }\end{array}$ & Host & Origin & $\begin{array}{l}\text { Date of } \\
\text { collection }\end{array}$ \\
\hline FJ001 & $V$. vinifera $c v$. Jingyu & Xingyuan, Zhengfang, Shunchang, Fujian & 2015.07 .07 \\
\hline FJ002 & V. vinifera $c v$. Beauty Finger & Xingyuan, Zhengfang, Shunchang, Fujian & 2015.07.07 \\
\hline FJ005 & $\begin{array}{l}\text { V. labruscana cv. Summer } \\
\text { Black }\end{array}$ & Xinjie, Yangxi, Meilie, Sanming, Fujian & 2015.07.07 \\
\hline FJ008 & $\begin{array}{l}\text { V. labruscana } c v \text {. Summer } \\
\text { Black }\end{array}$ & Xinjie, Yangxi, Meilie, Sanming, Fujian & 2015.07.07 \\
\hline FJ010 & V. vinifera $c v$. Kyoho & Beishan, Xibing, Fu'an, Fujian & 2015.07 .13 \\
\hline FJ011 & V. vinifera $c v$. Kyoho & Beishan, Xibing, Fu'an, Fujian & 2015.07 .13 \\
\hline FJ012 & V. vinifera $c v$. Kyoho & Beishan, Xibing, Fu'an, Fujian & 2015.07.13 \\
\hline FJ014 & V. vinifera $c v$. Kyoho & Beishan, Xibing, Fu'an, Fujian & 2015.07.13 \\
\hline FJ015 & V. vinifera $c v$. Kyoho & Beishan, Xibing, Fu'an, Fujian & 2015.07 .13 \\
\hline FJ016 & V. vinifera $c v$. Kyoho & Beishan, Xibing, Fu'an, Fujian & 2015.07.13 \\
\hline FJ017 & V. vinifera $c v$. Kyoho & Beishan, Xibing, Fu'an, Fujian & 2015.07.13 \\
\hline FJ018 & V. vinifera $c v$. Kyoho & Beishan, Xibing, Fu'an, Fujian & 2015.07 .13 \\
\hline FJ037 & V. vinifera $c v$. Kyoho & Beishan, Xibing, Fu'an, Fujian & 2015.07.10 \\
\hline FJ038 & V. vinifera $c v$. Kyoho & Beishan, Xibing, Fu'an, Fujian & 2015.07.10 \\
\hline FJ041 & V. vinifera $c v$. Kyoho & Xianghuan, Saiqi, Fu'an, Fujian & 2015.07.10 \\
\hline FJ043 & V. vinifera $c v$. Kyoho & Xianghuan, Saiqi, Fu'an, Fujian & 2015.07.10 \\
\hline FJ044 & V. vinifera $c$. Kyoho & Xianghuan, Saiqi, Fu'an, Fujian & 2015.07.10 \\
\hline FJ045 & V. vinifera $c v$. Kyoho & Xianghuan, Saiqi, Fu'an, Fujian & 2015.07.10 \\
\hline FJ047 & V. vinifera $c v$. Kyoho & Xianghuan, Saiqi, Fu'an, Fujian & 2015.07.10 \\
\hline FJ050 & $\begin{array}{l}\text { V. labruscana cv. Summer } \\
\text { Black }\end{array}$ & Xianghuan, Saiqi, Fu'an, Fujian & 2015.07.10 \\
\hline FJ051 & $\begin{array}{l}\text { V. labruscana } c v \text {. Summer } \\
\text { Black }\end{array}$ & Xianghuan, Saiqi, Fu'an, Fujian & 2015.07.10 \\
\hline FJ053 & $\begin{array}{l}\text { V. labruscana cv. Summer } \\
\text { Black }\end{array}$ & Xianghuan, Saiqi, Fu'an, Fujian & 2015.07.10 \\
\hline FJ055 & $\begin{array}{l}\text { V. labruscana } c v \text {. Summer } \\
\text { Black }\end{array}$ & Xianghuan, Saiqi, Fu'an, Fujian & 2015.07 .10 \\
\hline FJ056 & $\begin{array}{l}\text { V. labruscana } c v \text {. Summer } \\
\text { Black }\end{array}$ & Xianghuan, Saiqi, Fu'an, Fujian & 2015.07.10 \\
\hline FJ058 & $\begin{array}{l}\text { V. labruscana } c v \text {. Summer } \\
\text { Black }\end{array}$ & Xianghuan, Saiqi, Fu'an, Fujian & 2015.07 .10 \\
\hline FJ059 & $\begin{array}{l}\text { V. labruscana } c v \text {. Summer } \\
\text { Black }\end{array}$ & Xianghuan, Saiqi, Fu'an, Fujian & 2015.07.10 \\
\hline FJ060 & $\begin{array}{l}\text { V. labruscana } c v \text {. Summer } \\
\text { Black }\end{array}$ & Xianghuan, Saiqi, Fu'an, Fujian & 2015.07.10 \\
\hline FJ061 & $\begin{array}{l}\text { V. labruscana cv. Summer } \\
\text { Black }\end{array}$ & Xianghuan, Saiqi, Fu'an, Fujian & 2015.07.10 \\
\hline FJ062 & $\begin{array}{l}\text { V. labruscana cv. Summer } \\
\text { Black }\end{array}$ & Xianghuan, Saiqi, Fu'an, Fujian & 2015.07.10 \\
\hline FJ063 & $\begin{array}{l}\text { V. labruscana cv. Summer } \\
\text { Black }\end{array}$ & Xianghuan, Saiqi, Fu'an, Fujian & 2015.07.10 \\
\hline FJ064 & $\begin{array}{l}\text { V. labruscana } c v \text {. Summer } \\
\text { Black }\end{array}$ & Xianghuan, Saiqi, Fu'an, Fujian & 2015.07.10 \\
\hline FJ067 & $\begin{array}{l}\text { V. labruscana } c v \text {. Summer } \\
\text { Black }\end{array}$ & Houfang, Mayangxi, Changtai, Fujian & 2015.07.11 \\
\hline FJ068 & $\begin{array}{l}\text { V. labruscana cv. Summer } \\
\text { Black }\end{array}$ & Houfang, Mayangxi, Changtai, Fujian & 2015.07.11 \\
\hline FJ069 & $\begin{array}{l}\text { V. labruscana } c v \text {. Summer } \\
\text { Black }\end{array}$ & Houfang, Mayangxi, Changtai, Fujian & 2015.07.11 \\
\hline
\end{tabular}




\begin{tabular}{|c|c|c|c|}
\hline $\begin{array}{l}\text { Isolate } \\
\text { no. }\end{array}$ & Host & Origin & $\begin{array}{l}\text { Date of } \\
\text { collection }\end{array}$ \\
\hline FJ071 & V. vinifera 'Rose red' & Houfang, Mayangxi, Changtai, Fujian & 2015.07 .11 \\
\hline FJ072 & $\begin{array}{l}\text { V. vinifera 'Shennong gold } \\
\text { queen' }\end{array}$ & Pudang, Xindian, Jin’an, Fuzhou, Fujian & 2015.07.13 \\
\hline FJ073 & $\begin{array}{l}\text { V. vinifera 'Shennong gold } \\
\text { queen' }\end{array}$ & Pudang, Xindian, Jin'an, Fuzhou, Fujian & 2015.07.13 \\
\hline FJ074 & $\begin{array}{l}\text { V. vinifera 'Shennong gold } \\
\text { queen' }\end{array}$ & Pudang, Xindian, Jin’an, Fuzhou, Fujian & 2015.07.13 \\
\hline FJ080 & $\begin{array}{l}\text { V. vinifera 'Shennong gold } \\
\text { queen' }\end{array}$ & Pudang, Xindian, Jin’an, Fuzhou, Fujian & 2015.07.13 \\
\hline FJ083 & $\begin{array}{l}V \text {. vinifera 'Shennong gold } \\
\text { queen' }\end{array}$ & Pudang, Xindian, Jin'an, Fuzhou, Fujian & 2015.07.13 \\
\hline GZ012 & V. vinifera 'Crystal' & Xinlian, Jiaoli, Sandu, Guizhou & 2015.07.19 \\
\hline GZ014 & V. vinifera 'Crystal' & Xinlian, Jiaoli, Sandu, Guizhou & 2015.07.19 \\
\hline GZ019 & V. vinifera 'Crystal' & $\begin{array}{l}\text { Wenquan, Hailong, Honghuagang, Zunyi, } \\
\text { Guizhou }\end{array}$ & 2015.07.19 \\
\hline GZ020 & V. vinifera 'Crystal' & $\begin{array}{l}\text { Wenquan, Hailong, Honghuagang, Zunyi, } \\
\text { Guizhou }\end{array}$ & 2015.07.20 \\
\hline GZ024 & V. vinifera 'Crystal' & $\begin{array}{l}\text { Wenquan, Hailong, Honghuagang, Zunyi, } \\
\text { Guizhou }\end{array}$ & 2015.07.20 \\
\hline GZ025 & V. vinifera 'Crystal' & $\begin{array}{l}\text { Grape base of Guizhou University, Huaxi, } \\
\text { Guiyang, Guizhou }\end{array}$ & 2015.07.21 \\
\hline GZ026 & V. vinifera 'Crystal' & $\begin{array}{l}\text { Grape base of Guizhou University, Huaxi, } \\
\text { Guiyang, Guizhou }\end{array}$ & 2015.07.21 \\
\hline GZ029 & V. vinifera 'Crystal' & $\begin{array}{l}\text { Grape base of Guizhou University, Huaxi, } \\
\text { Guiyang, Guizhou }\end{array}$ & 2015.07.21 \\
\hline GZ030 & V. vinifera 'Crystal' & $\begin{array}{l}\text { Grape base of Guizhou University, Huaxi, } \\
\text { Guiyang, Guizhou }\end{array}$ & 2015.07.21 \\
\hline GZ033 & V. vinifera 'Crystal' & $\begin{array}{l}\text { Grape base of Guizhou University, Huaxi, } \\
\text { Guiyang, Guizhou }\end{array}$ & 2015.07.21 \\
\hline
\end{tabular}

\section{Molecular characterization}

Total genomic DNA was extracted from 5-7-day-old mycelia according to the method of Damm et al. (2012), and then used as a template after the detection by the electrophoresis (Bio-Rad gel imaging GelDoc XR + system, Bio-Rad, Hercules, CA, USA). Specific primers were used for PCR amplification of the 5.8S nuclear ribosomal gene with the two flanking internal transcribed spacers (ITS), a 200-bp intron of the glyceraldehyde-3-phosphate dehydrogenase (GAPDH), partial sequences of the chitin synthase 1 (CHS-1), actin (ACT) and $\beta$-tubulin (TUB2) genes were amplified using primer pairs ITS1/ITS4 (White et al. 1990), GDF/GDR (Templeton et al. 1992), CHS79F/CHS345R (Carbone \& Kohn 1999), ACT512F/ACT783R (Carbone \& Kohn 1999) and BT1/BT2 (O’Donnell \& Cigelnik 1997) respectively. PCR was performed on a Bio-Rad PCR machine (Applied Bio-systems, VERITI Thermal Cycler) in a total volume of $25 \mu \mathrm{L}: 2 \times$ Taq PCR Master Mix $12.5 \mu \mathrm{L}$, DNA template $1 \mu \mathrm{L}$, each primer $1 \mu \mathrm{L}$ and sterile double distilled water 9.5 $\mu \mathrm{L}$. The PCR amplification procedure was performed as follows: $95{ }^{\circ} \mathrm{C}$ denaturation for $4 \mathrm{~min}$, $95{ }^{\circ} \mathrm{C}$ denaturation $30 \mathrm{~s}, 56{ }^{\circ} \mathrm{C}$ (ACT and TUB), $59{ }^{\circ} \mathrm{C}$ (CHS and GADPH), $57{ }^{\circ} \mathrm{C}$ (ITS) annealing $30 \mathrm{~s}, 72{ }^{\circ} \mathrm{C}$ extension of $1 \mathrm{~min}, 35$ amplification cycles, and $72{ }^{\circ} \mathrm{C}$ extension for $7 \mathrm{~min}$. The PCR products were purified by $1.2 \%$ agarose gel electrophoresis and then sequenced by Biomed Company, Beijing, China. After analysis by NCBI BLAST, the results were submitted to the GenBank database and the corresponding accession number was obtained (Table 2). 
Table 2 Strains of Colletotrichum spp. studied, with collection details and GenBank accessions.

\begin{tabular}{|c|c|c|c|c|c|c|}
\hline \multirow{2}{*}{ Species } & \multirow{2}{*}{ Strain no. } & \multicolumn{5}{|c|}{ Genbank accession Numbers } \\
\hline & & ITS & BUT & ACT & CHS & GAPDH \\
\hline C. aenigma & JZB330035 & 156870 & KF288980 & KF377502 & KF289014 & KF377502 \\
\hline C. aeschynomenes & ICMP17673 & JX010176 & JX010392 & JX009483 & JX009799 & JX009930 \\
\hline C. alatae & ICMP12071 & JX010190 & JX010383 & JX009471 & JX009837 & JX009990 \\
\hline C. alienum & ICMP12071 & JX010251 & JX010411 & JX009572 & JX009882 & JX010028 \\
\hline C. aotearoa & ICMP18537 & JX010205 & JX010420 & JX009564 & JX009853 & JX010005 \\
\hline C. asianum & ICMP18580 & FJ972612 & JX010406 & JX009584 & JX009867 & JX010053 \\
\hline C. boninense & CBS123755 & JQ005153 & JQ005588 & JQ005327 & JQ005414 & JQ005327 \\
\hline C. citri & GZ019 & KX670382 & KX756974 & KX756972 & KX687652 & KX687654 \\
\hline C. clidemiae & ICMP18658 & JX010265 & JX010438 & JX009537 & JX009877 & JX009989 \\
\hline C. cordylinicola & ICMP18579 & JX010226 & JX010440 & HM470235 & JX009864 & JX009975 \\
\hline C. dianesei & CMM4083 & KC329779 & KC517254 & KC517298 & - & КC329779 \\
\hline C. endophytica & LC0324 & KC633854 & - & KF306258 & - & KC832854 \\
\hline C. fructicola & ICMP18581 & JX010165 & JX010405 & FJ907426 & JX009866 & JX010033 \\
\hline C. fructivorum & Coll414 & JX145145 & JX145196 & - & - & - \\
\hline C. grevilleae & CBS132879 & KC297078 & KC297102 & KC296941 & KC297056 & KC297010 \\
\hline C. gloeosporioides & CBS1 & JQ005 & JQ005587 & JQ005500 & JQ005326 & JQ005239 \\
\hline C. hebeiense & JZB330028 & KF156863 & KF288975 & KF377532 & KF289008 & KF377495 \\
\hline C. horri & ICMP10492 & GQ329690 & JX010450 & JX009438 & JX009752 & GQ329681 \\
\hline C. kahawae & ICMP17816 & JX010231 & JX010444 & JX009452 & JX009813 & JX010012 \\
\hline C. murrayae & GZAAS5.0950 & JQ247633 & JQ247644 & JQ247657 & - & JQ247657 \\
\hline musae & ICMP19 & 6 & HQ5 & $\mathrm{JX}$ & & 50 \\
\hline C. nupharicola & CBS & $\mathrm{JX}$ & JX01 & $\mathrm{JX}$ & JXOC & 972 \\
\hline Colletotrichum sp. & FJ074 & KX670383 & KX756975 & KX756973 & KX687653 & KX687655 \\
\hline C. proteae & CBS132882 & KC297079 & KC297101 & KC296940 & KC296986 & KC297009 \\
\hline C. psidii & CBS145.29 & JX010219 & JX010443 & JX009515 & JX009901 & JX009967 \\
\hline C. queenslandicum & ICMP1778 & JX010276 & JX010414 & JX009447 & JX009899 & JX009934 \\
\hline C. rhexiae & Coll 1026 & JX145128 & JX145179 & - & - & - \\
\hline C. salsolae & ICMP19051 & JX010242 & JX010403 & JX009562 & JX009863 & JX009916 \\
\hline C. siamense & ICMP18578 & JX010171 & JX010404 & FJ907423 & JX009865 & JX009924 \\
\hline C. syzygicola & DNCL021 & KF242094 & KF254880 & KF157801 & - & KF242156 \\
\hline C. temperatum & Coll 883 & JX145159 & JX145211 & - & - & - \\
\hline C. theobromicola & ICMP18649 & JX010294 & JX010447 & JX009444 & JX009869 & JX010006 \\
\hline C. $t i$ & ICMP 4832 & JX010269 & JX010442 & JX009520 & JX009898 & JX009952 \\
\hline C. tropicale & ICMP 18653 & JX010264 & JX010407 & JX01407 & JX009870 & JX010007 \\
\hline C. viniferum & JZB330001 & KF156840 & KF288965 & KF377506 & KF288982 & KF377469 \\
\hline C. viniferum & FJ001 & KX594332 & KX621321 & KX621369 & KX687604 & KX687656 \\
\hline C. viniferum & FJ002 & KX594333 & KX621322 & KX761398 & KX687605 & KX687657 \\
\hline C. viniferum & FJ005 & KX594334 & KX621323 & KX621370 & KX687606 & KX687658 \\
\hline C. viniferum & & KX594335 & KX621324 & KX621371 & KX687607 & KX687659 \\
\hline C. viniferum & FJ010 & KX594336 & KX621325 & KX621372 & KX687608 & KX687660 \\
\hline C. viniferum & FJ011 & KX594337 & KX621326 & KX621373 & KX687609 & KX687661 \\
\hline
\end{tabular}




\begin{tabular}{|c|c|c|c|c|c|c|}
\hline \multirow{2}{*}{ Species } & \multirow{2}{*}{ Strain no. } & \multicolumn{5}{|c|}{ Genbank accession Numbers } \\
\hline & & ITS & BUT & ACT & CHS & GAPDH \\
\hline C. viniferum & FJ012 & KX594338 & KX621327 & KX621374 & KX687610 & KX687662 \\
\hline C. viniferum & FJ014 & KX594339 & KX621328 & KX621375 & KX687611 & KX687663 \\
\hline C. viniferum & FJ015 & KX594340 & KX621329 & KX621376 & KX687612 & KX687664 \\
\hline C. viniferum & FJ016 & KX594341 & KX621330 & KX621377 & KX687613 & KX687665 \\
\hline C. viniferum & FJ017 & KX594342 & KX621331 & KX621378 & KX687614 & KX687666 \\
\hline C. viniferum & FJ018 & KX594343 & KX621332 & KX621379 & KX687615 & KX687667 \\
\hline C. viniferum & FJ037 & KX594344 & KX621333 & KX621380 & KX687616 & KX687668 \\
\hline C. viniferum & FJ038 & KX594345 & KX621334 & KX621381 & KX687617 & KX687669 \\
\hline C. viniferum & FJ041 & KX594346 & KX621335 & KX621382 & KX687618 & KX687670 \\
\hline C. viniferum & FJ043 & KX594347 & KX621336 & KX621383 & KX687619 & KX687671 \\
\hline C. viniferum & FJ044 & KX594348 & KX621337 & KX621384 & KX687620 & KX687672 \\
\hline C. viniferum & FJ045 & KX594349 & KX621338 & KX621385 & KX687621 & KX687673 \\
\hline C. viniferum & FJ047 & KX594350 & KX621339 & KX621386 & KX687622 & KX687674 \\
\hline C. viniferum & FJ050 & KX594351 & KX621340 & KX621387 & KX687623 & KX687675 \\
\hline C. viniferum & FJ051 & KX594352 & KX621341 & KX621388 & KX687624 & KX687676 \\
\hline C. viniferum & FJ053 & KX594353 & KX621342 & KX621389 & KX687625 & KX687677 \\
\hline C. viniferum & FJ055 & KX594354 & KX621343 & KX621390 & KX687626 & KX687678 \\
\hline C. viniferum & FJ056 & KX594355 & KX621344 & KX621391 & KX687627 & KX687679 \\
\hline C. viniferum & FJ058 & KX594356 & KX621345 & KX621392 & KX687628 & KX687680 \\
\hline C. viniferum & FJ059 & KX594357 & KX621346 & KX621393 & KX687629 & KX687681 \\
\hline C. viniferum & FJ060 & KX594358 & KX621347 & KX621394 & KX687630 & KX687682 \\
\hline C. viniferum & FJ061 & KX594359 & KX621348 & KX621395 & KX687631 & KX687683 \\
\hline C. viniferum & FJ062 & KX594360 & KX621349 & KX621396 & KX687632 & KX687684 \\
\hline C. viniferum & FJ063 & KX594361 & KX621350 & KX621397 & KX687633 & KX687685 \\
\hline C. viniferum & FJ064 & KX594362 & KX621351 & KX621398 & KX687634 & KX687686 \\
\hline C. viniferum & FJ067 & KX594363 & KX621352 & KX621399 & KX687635 & KX687687 \\
\hline C. viniferum & FJ068 & KX594364 & KX621353 & KX621400 & KX687636 & KX687688 \\
\hline C. viniferum & FJ069 & KX594365 & KX621354 & KX621401 & KX687637 & KX687689 \\
\hline C. viniferum & FJ071 & KX594366 & KX621355 & KX621402 & KX687638 & KX687690 \\
\hline C. viniferum & FJ072 & KX594367 & KX621356 & KX621403 & KX687639 & KX687691 \\
\hline C. viniferum & FJ073 & KX594368 & KX621357 & KX621404 & KX687640 & KX687692 \\
\hline C. viniferum & FJ080 & KX594369 & KX621358 & KX621405 & KX687641 & KX687693 \\
\hline C. viniferum & FJ083 & KX594370 & KX621359 & KX621406 & KX687642 & KX687694 \\
\hline C. viniferum & GZ012 & KX594371 & KX621360 & KX621407 & KX687643 & KX687695 \\
\hline C. viniferum & GZ014 & KX594372 & KX621361 & KX621408 & KX687644 & KX687696 \\
\hline C. viniferum & GZ020 & KX594373 & KX621362 & KX621409 & KX687645 & KX687697 \\
\hline C. viniferum & GZ024 & KX594374 & KX621363 & KX621410 & KX687646 & KX687698 \\
\hline C. viniferum & GZ025 & KX594375 & KX621364 & KX621411 & KX687647 & KX687699 \\
\hline C. viniferum & GZ026 & KX594376 & KX621365 & KX621412 & KX687648 & KX687700 \\
\hline C. viniferum & GZ029 & KX594377 & KX621366 & KX621413 & KX687649 & KX687701 \\
\hline C. viniferum & GZ030 & KX594378 & KX621367 & KX621414 & KX687650 & KX687702 \\
\hline C. viniferum & GZ033 & KX594379 & KX621368 & KX621415 & KX687651 & KX687703 \\
\hline
\end{tabular}




\section{Phylogenetics analysis}

Single gene regions were aligned using Clustal X1.81 (Thompson et al. 1997) and combined using BioEdit v.7.0.9.0 (Hall 1999). Further alignment of the sequences was done using the default settings of MAFFT v.7 (Katoh \& Toh 2008; http://mafft.cbrc.jp/alignment/server/) and manual adjustment was conducted using BioEdit, where necessary. Maximum parsimony analysis was performed using phylogenetic analysis using parsimony v. $4.0 \mathrm{~b} 10$ (Swofford 2002) to obtain the most parsimonious trees. Gaps were treated as missing data and ambiguously aligned regions were excluded. Trees were inferred using the heuristic search option with tree-bisection-reconnection branch swapping and 1,000 random sequence additions. Maxtrees were set up to 5,000, branches of zero length were collapsed, and all multiple parsimonious trees were saved. Descriptive tree statistics for parsimony (tree length, consistency index, retention index, rescaled consistency index, and homoplasy index) were calculated for trees generated under different optimality criteria. The robustness of the most parsimonious trees was evaluated by 1,000 bootstrap replications resulting from the maximum parsimony analysis (Hillis \& Bull 1993). Kishino-Hasegawa tests (Kishino \& Hasegawa 1989) were performed in order to determine whether trees were significantly different.

Bayesian inference was used in addition to construct the phylogenies using Mr. Bayes v.3.1.2 (Ronquist \& Huelsenbeck 2003). MrModeltest v. 2.3 (Nylander 2004) was used to carry out statistical selection of the best-fit model of nucleotide substitution and was incorporated into the analysis. Six simultaneous Markov chains were run for 1,000,000 generations, and trees were sampled every $100^{\text {th }}$ generation. The 2,000 trees representing the burn-in phase of the analyses were discarded, and the remaining 8,000 trees were used for calculating posterior probabilities in the majority rule consensus tree.

\section{Pathogenicity testing}

In order to verify the source and pathogenicity of the strains, the grapevine surface was disinfected with $70 \%$ alcohol for $1 \mathrm{~min}$, washed three times with sterile water. Every seven grapes were placed in a 9-cm petri dish, and every five dishes were considered as a group. A total of four groups were used in this study, namely CK (control), C. citri, C. viniferum and Colletotrichum sp. strain FJ074. Superficial wounds in the grape epidermis were carried out with a sterilized insect pin. Four-mm-diameter disks of PDA were removed with a puncher and then placed on the wound. The grape surface with sterile PDA was used as a negative control. Each petri dish was incubated at $25{ }^{\circ} \mathrm{C}$ with $80 \%$ humidity. After 48 hours, the PDA disks were removed, and the pathogenesis situation of grapevine was observed after 7-10 days incubation.

\section{Results}

\section{Pathogen morphology}

The 50 strains used in this study were isolated from diseased grapevine fruits with typical ripe rot symptoms (Fig. 1) in Nanping, Sanming, Ningde, Zhangzhou, and Zunyi, and then separated into three types according to the morphology, color, and other characteristics. The three types were: Type A: the appearance of colonies was round or nearly round, mycelia appeared light gray, while the bottom was orange and there was no black generation, aerial mycelia were white and fluffy but no obvious stripes were seen (FJ074) (Fig. 3); Type B: colonies were also circular or nearly round, mycelia were off-white and distributed as stripes, melanin can be seen clearly in the center of the bottom, while the growth was slow and the white aerial mycelium were arranged densely (GZ019) (Fig. 4); Type C: colonies were round or nearly round, mycelia were brown and melanin was obvious in the bottom, the gray aerial mycelium were represented as flocculence and were arranged irregularly (FJ017) (Fig. 5). As shown in Table 3, the pycnidia and conidia were significantly different among the three types, the largest of which was FJ074, with a length and width of $191.301 \mu \mathrm{m}$ and $237.728 \mu \mathrm{m}$, respectively. 
Table 3 Synopsis of morphological data of Colletotrichum species in grape from this study.

\begin{tabular}{llllll}
\hline $\begin{array}{l}\text { Species and isolate } \\
\text { number }\end{array}$ & \multicolumn{2}{l}{ Conidioma } & & Conidia & \\
\cline { 2 - 3 } \cline { 5 - 6 } & length $(\boldsymbol{\mu m})$ & Width $(\boldsymbol{\mu m})$ & & length $(\mu \mathbf{m})$ & width $(\boldsymbol{\mu m})$ \\
\hline FJ017 & $180.953( \pm 9.364)$ & $165.865( \pm 15.144)$ & & $14.677( \pm 1.282)$ & $4.954( \pm 0.430)$ \\
FJ074 & $238.728( \pm 28.835)$ & $191.301( \pm 39.516)$ & & $15.166( \pm 2.485)$ & $5.232( \pm 0.673)$ \\
GZ019 & $162.328( \pm 23.490)$ & $163.359( \pm 20.552)$ & & $9.045( \pm 1.153)$ & $4.135( \pm 0.475)$ \\
\hline
\end{tabular}
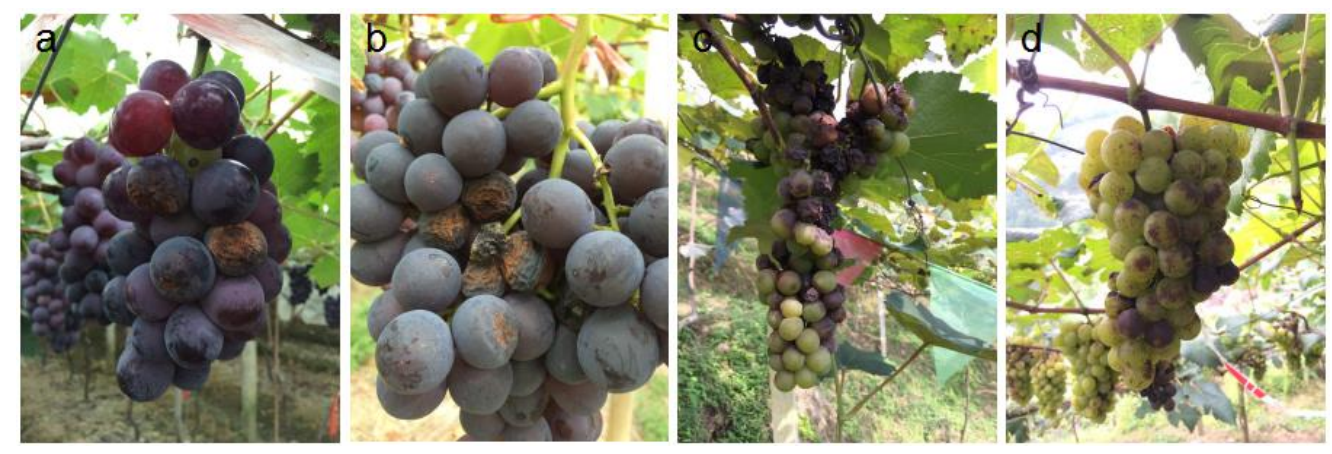

Fig. 1 - Symptoms of the ripe rot disease found in vineyards $\mathbf{a}-\mathbf{b}$ Grape fruits in Fujian. c-d Grape fruits in Guizhou.

\section{Phylogenetic analysis}

Phylogenetic analyses were carried out using combined ACT, GADPH, CHS, TUB2, and ITS sequences from 121 Colletotrichum strains with Moniochaetes infuscans as the outgroup taxa. As Fig. 2 shows, with the exception of strains FJ074 and GZ019, the other 48 strains were all identified as $C$. viniferum. Strain GZ019 was clustered with $C$. citri with a $100 \%$ bootstrap value. The strain of FJ074 created a separate branch with a 100\% bootstrap value and is a sister taxon to $C$. cliviae. However, until further collections are made this species will be kept as Colletotrichum sp.

\section{Pathogenicity testing}

The pathogenicity of the three species of ripe rot showed that all of them had the ability to infect $V$. vinifera $c v$. Kyoho and cause the typical symptoms of ripe rot (Fig. 6). The species that we re-isolated from the infection site of grapes were identical with the test strain, which was in full compliance with Koch's postulates.

\section{Taxonomy}

Colletotrichum citri F. Huang, L. Cai, K.D. Hyde \& H.Y. Li

The species was isolated from V. vinifera 'Crystal' collected from Guizhou. This is the first report of this species causing grape ripe rot in the world. After incubated at $25^{\circ} \mathrm{C}$ for 6 days, the diameter of colony was $6.50 \mathrm{~cm}$. The round colony appeared to be gray on the positive, and gray to black on the back, melanin was visible. The size of the transparent conidia was $9.05 \times 4.14 \mu \mathrm{m}$, both ends were blunt round.

\section{Colletotrichum sp. strain FJ074}

Pathogen on $V$. vinifera. Colonies growing from single conidia on PDA grey, reverse orange-red in centre, off-white towards the edge, reaching a maximum of $80.0 \mathrm{~mm}$ diameter in 7 days at $28{ }^{\circ} \mathrm{C}$, growth rate $11.4 \mathrm{~mm} /$ day. Aerial mycelium was white, rarefaction, flocculence. Asexual morph developed on PDA. Conidiomata 210-267 $\mu$ m diammeter, abundant, black, oval, solitary to aggregated, with orange spore masses. Conidia 13.7-17.7 $\times 4.5-5.9(\bar{x}=15.2 \times 5.2, \mathrm{n}=$ 10) hyaline, smooth-walled, aseptate, both ends were sharp to cylindrical or clavate with rounded apices, contents granular and mostly present at the polar ends leaving a opaque region in the centre. Appressoria not observed. Sexual morph not observed. 


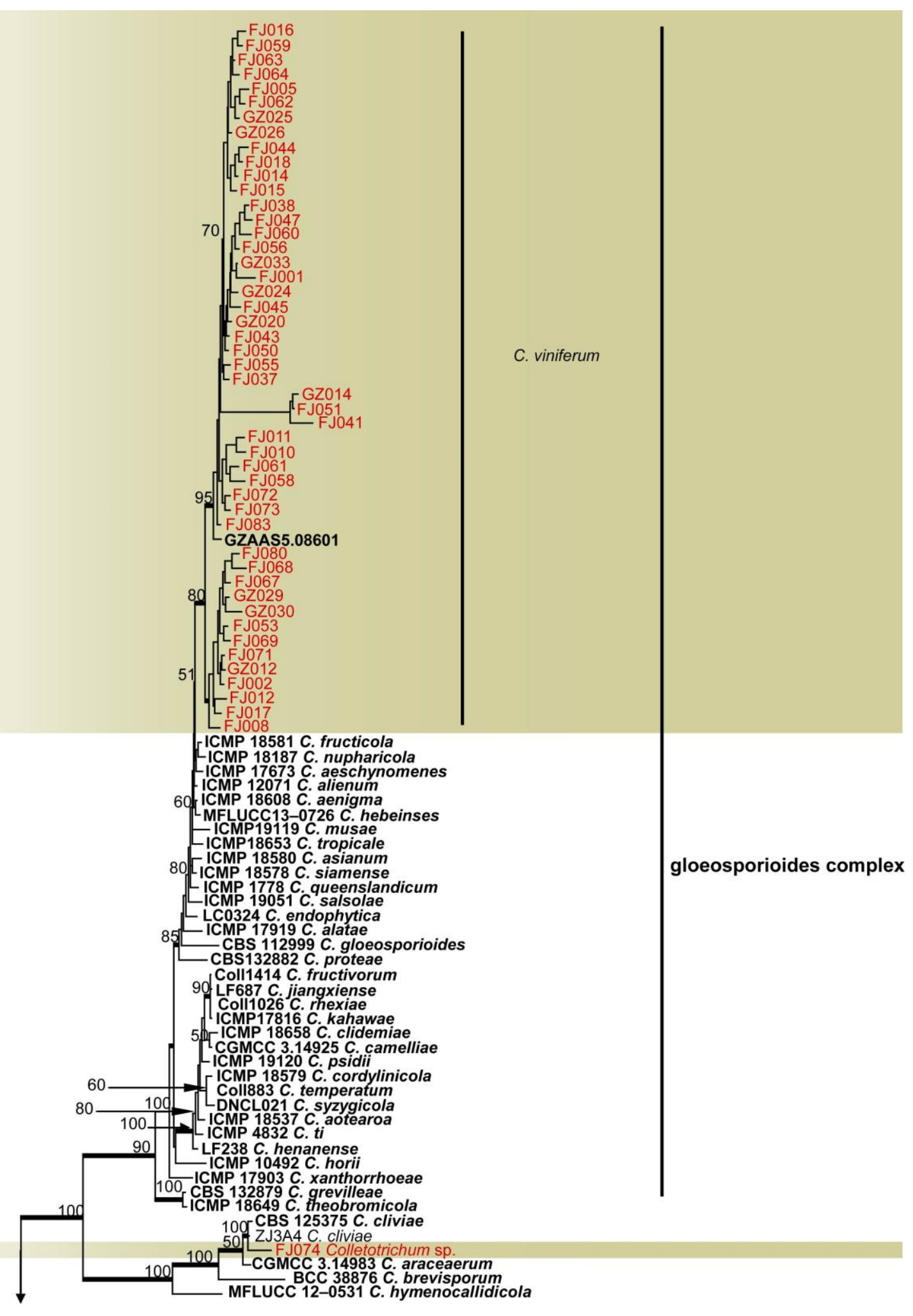

Fig. 2 - One of the 100 most parsimonious trees obtained from a heuristic search of combined ACT, $\beta$-tubulin, CHS-1, GAPDH and ITS sequenced data of Colletotrichum species. Parsimony bootstrap support values greater than $70 \%$ are indicated above the nodes and branches with Bayesian posterior probabilities above 0.90 are given in bold. The ex-type strains are in bold; isolates of this study are in red. The scale bar indicates ten changes. The tree is rooted with $M$. infuscans CBS 869.96. 


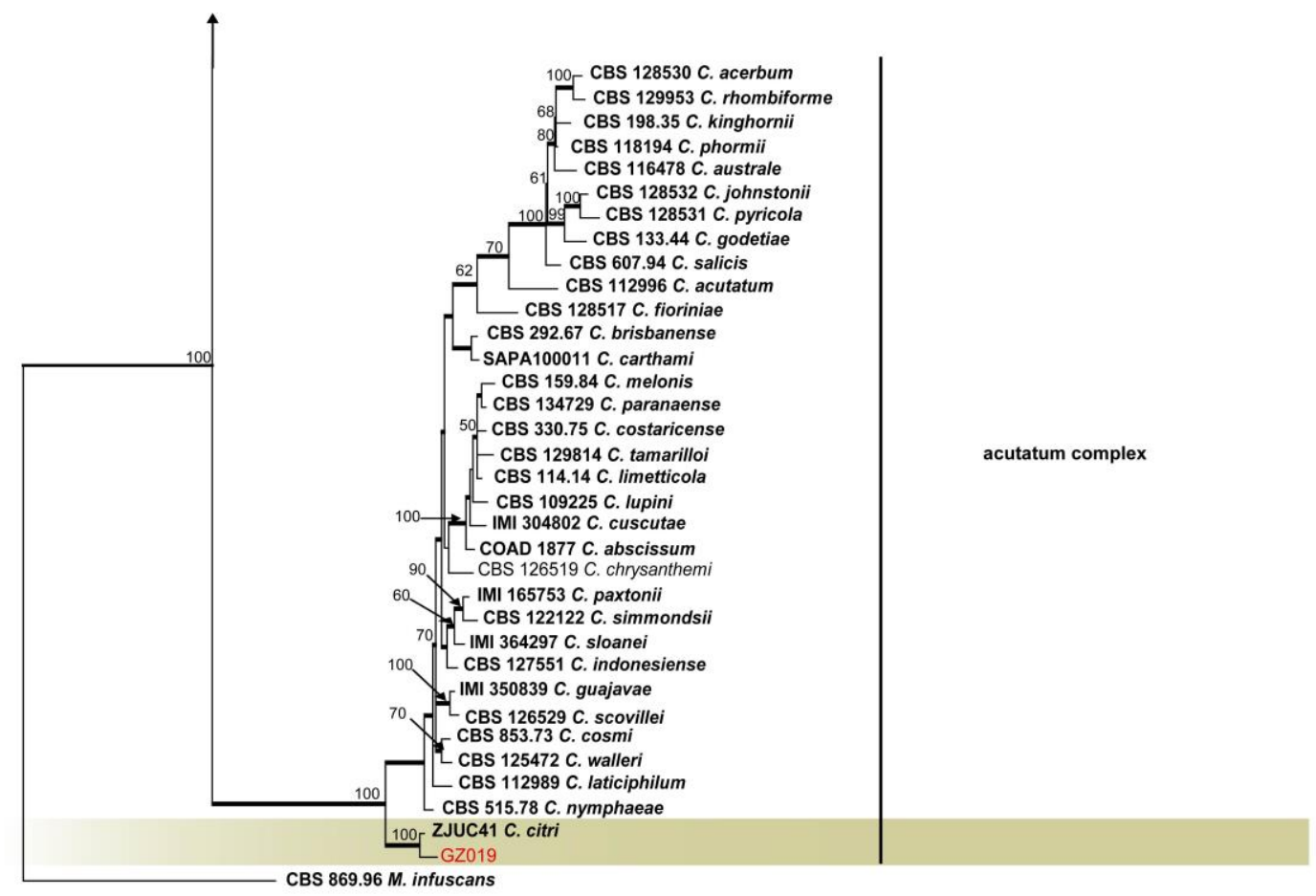

10
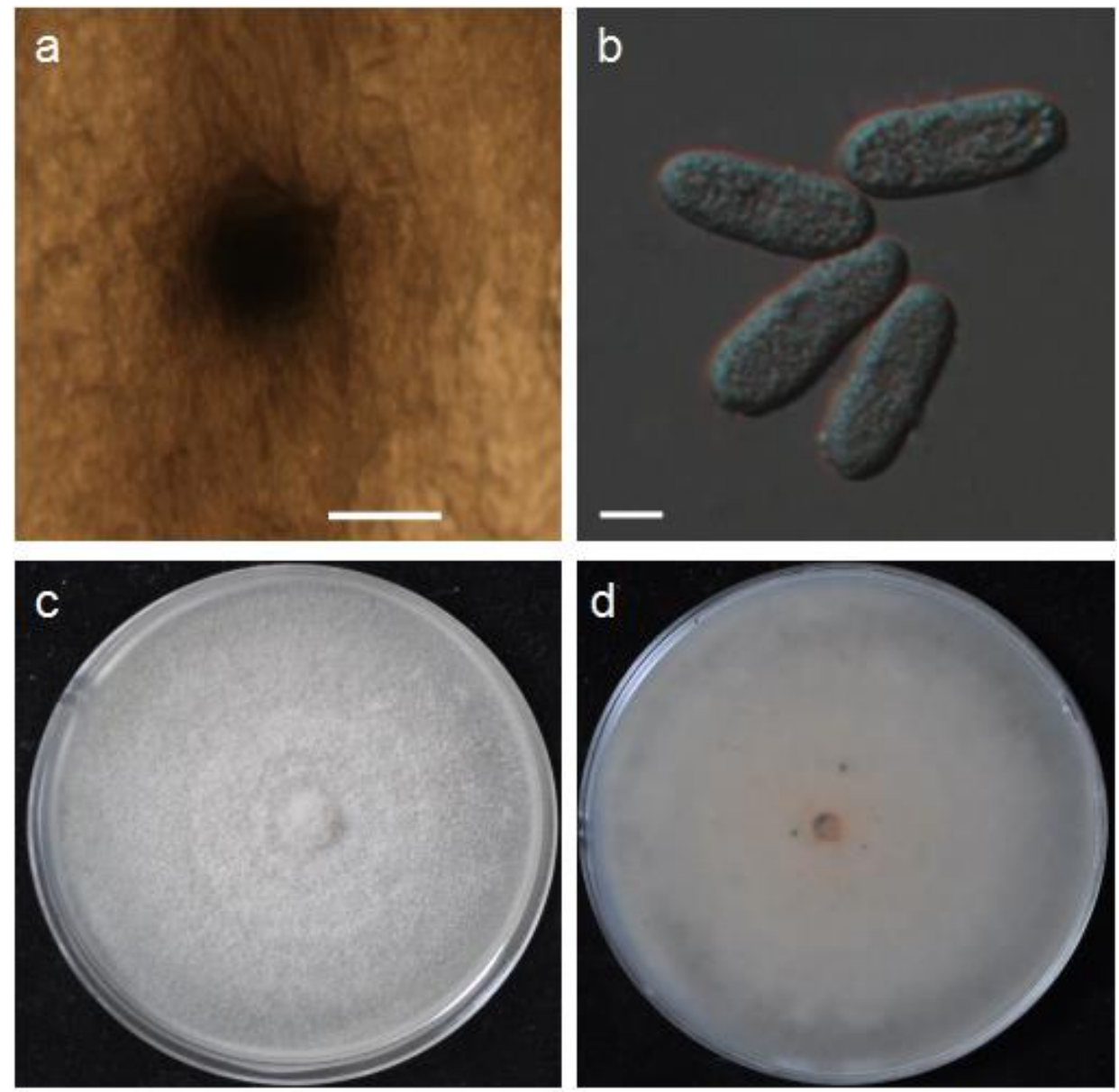

Fig. 3 - Colletotrichum sp. strain FJ074 a Conidioma on PDA. b Conidia. c Upper view of colony ( 7 days old). d Reverse view of colony ( 7 days old). Scale bars: $a=200 \mu \mathrm{m}, b=5 \mu \mathrm{m}$. 

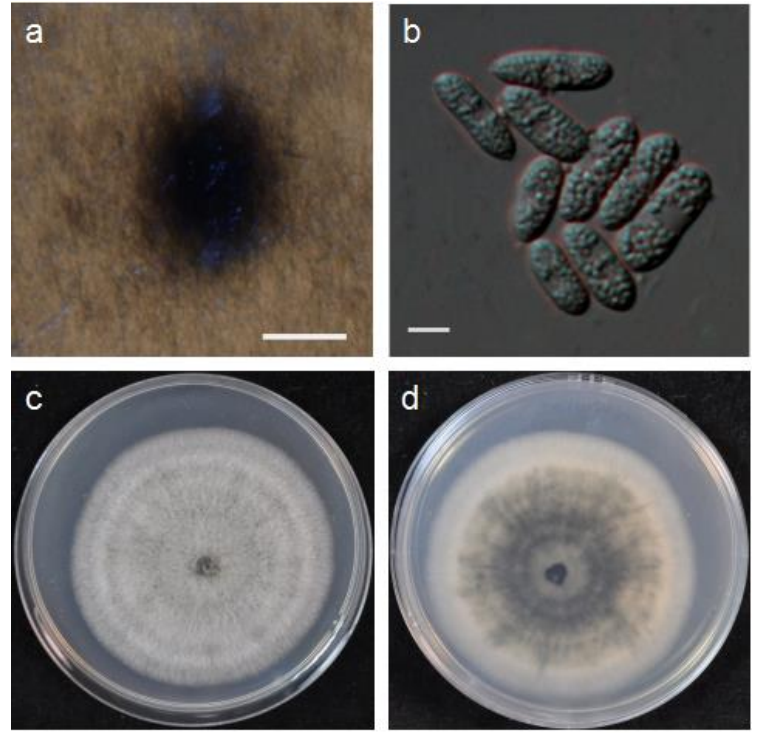

Fig. 4 - Colletotrichum citri a Conidioma on PDA. b Conidia. c Upper view of colony (7 days old). d Reverse view of colony ( 7 days old). Scale bars: $a=200 \mu \mathrm{m}, b=5 \mu \mathrm{m}$.
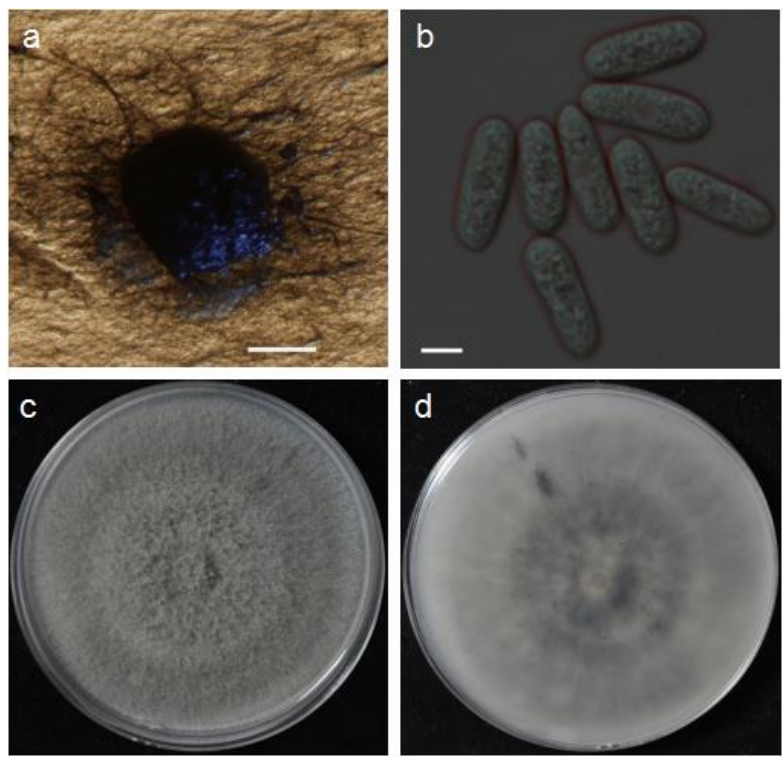

Fig. 5 - Colletotrichum viniferum a Conidioma on PDA. b Conidia. c Upper view of colony (7 days old). d Reverse view of colony (7 days old). Scale bars: $a=200 \mu \mathrm{m}, b=5 \mu \mathrm{m}$.
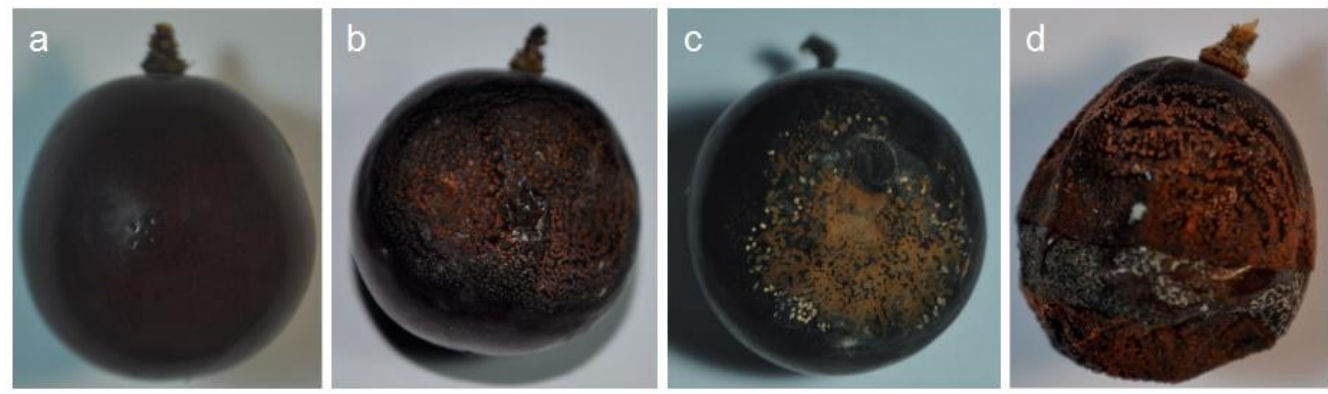

Fig. 6 - Pathogenesis situation of the grapevine a CK. b Colletotrichum sp.. c C. viniferum. d $C$. citri. 
Material examined: CHINA, Fujian, Fuzhou, Jin'an, Xindian Pudang, from V. vinifera 'Shennong gold queen', July 13, 2015, Lei yan.

Notes: This species was isolated from $V$. vinifera 'Shennong gold queen' in Fujian. After incubated at $25^{\circ} \mathrm{C}$ for 6 days, the colony was $8 \mathrm{~cm}$ in diameter. The appearance of colony was round and gray on the positive, off-white on the back. Hyphae were closely, and the orange conidiophores can be found on the bottom. The transparent conidia was $15.17 \times 5.23 \mu \mathrm{m}$ in dimension, both ends were blunt round. Based on multi-locus sequence data, this species is phylogenetically closely related to $C$. cliviae and $C$. araceaerum. However, until further collections are made we intend to keep this species as Colletotrichum sp.

Colletotrichum viniferum L.J. Peng, L. Cai, K.D. Hyde \& Zi Y. Ying

This species was isolated from 48 diseased grape samples with irregular lesions, including one $V$. vinifera $c v$. Jingyu, one $V$. vinifera $c v$. Beauty Finger, one $V$. vinifera 'Rose red', four $V$. vinifera 'Shennong gold queen', fifteen $V$. vinifera cv. Kyoho and seventeen $V$. Labruscana cv. Summer Black from Fujian, as well as nine V. vinifera 'Crystal' from Guizhou. Peng et al. (2013) first reported $C$. viniferum in China and described the morphological characteristics in detail. Phylogenetic analysis showed that 48 isolates of $C$. viniferum were clustered together, which is consistent with their similar morphological characteristics.

\section{Discussion}

Grape ripe rot is a serious disease that occurs on mature fruits or during the harvest season which damage to the yield and quality of grapes. The infected fruits turn dark brown and recessed, small black particles are distributed in a ring on the surface of the disease spots. Under high humidity conditions, a pink or orange conidia heap can be found on the surface, but it is difficult to distinguish the ripe rot species according to symptoms on leaves and fruits with visual observation. In a previous report, Colletotrichum gloeosporioides (Penz) was treated as the only ripe rot causing agent (Whitelaw et al. 2007), while with the progress of research, C. acutatum J.H. was identified to be the causal agent of grape ripe rot in Australia, Japan, South Korea and the United States (Hong et al. 2008). In fact, $C$. viniferum (Peng et al. 2013) as well as $C$. aenigma and $C$. hebeiense (Yan et al. 2015) have also been reported to be ripe rot pathogens.

In our study, 50 Colletotrichum isolates were isolated from diseased grape samples from Fujian and Guizhou Provinces. The strain FJ074 which isolated from Pudang, Xindian, Jin'an, Fuzhou, Fujian, is a Colletotrichum sp., whose colony morphology was significantly different with the other strains (Figs. 3-5), and pycnidia as well as conidia were obviously larger than $C$. viniferum (Table 3). Multi-gene analysis showed that Colletotrichum sp. and C. cliviae were in the same cluster. Chowdappa et al. (2014) has shown that $C$. cliviae led to anthracnose on Cymbidium dendulum in India. The colonies appeared olive gray on the front and dark green on the back and had cylindrical conidia harboring two blunt ends, but were a little bit bent. The size was 17.2 and $5.5 \mu \mathrm{m}$, respectively, with the appressorium cracked and shaped irregularly. The strain $C$. citri (GZ019), isolated from Wenquan, Hailong, Honghuagang, Zunyi, Guizhou, was a new Colletotrichum sp. found in the citrus (Huang et al. 2013), our study is the first time to show the ripe rot on grapevine caused by $C$. citri, indicating that this species has a wide host range. Multi-gene analysis showed that except for the strains of Colletotrichum sp. and C. citri, all the other strains belonged to $C$. viniferum, while the genotypes were different. Yan et al. (2015) mentioned that $C$. viniferum has a divergence in phylogeny. Silva et al. (2012) mentioned that the method of 'powerful genes' to identify anthracnose species is useful, but until now, mycologists have not reached a consensus on which marker genes should be used for the classification and identification of Colletotrichum species (Doyle et al. 2013).

The purpose of our study is to identify the pathogens of grape ripe rot in Fujian and Guizhou by both morphological and molecular biology methods, which will be helpful in further study of species dynamics and the distribution of grape ripe rot in China, and provide a theoretical 
basis for the prevention of diseases in the field. The results of our study show that grape ripe rot in Fujian Province and Guizhou Province is mainly due to $C$. viniferum, but not to $C$. gloeosporioides or C. acutatum, which consistent with Yan et al. (2015). More importantly, Colletotrichum sp. strain FJ074 is a new strain of causing grape ripe rot in Fujian Province, and C. citri is a new record for causing grape ripe rot.

\section{Acknowledgements}

Our study was supported by the earmarked fund for China Agriculture Research System (CARS-30), Special case of non-profit research institutes in Fujian Province (2016R1013-10) and Innovation Fund for Young Talents in Fujian Academy of Agricultural Sciences (YC2016-2).

\section{References}

Bailey JA, Jeger MJ. 1992 - Colletotrichum: biology, pathology and control. Mycologia 85.

Cai L, Hyde KD, Taylor PWJ, Weir BS, Waller J, Abang MM, Zhang JZ, Yang YL, Phoulivong S, Liu ZY, Prihastuti H, Shivas RG, McKenzie EHC, Johnston PR. 2009 - A polyphasic approach for studying Colletotrichum. Fungal Diversity 39, 183-204.

Carbone I, Kohn LM. 1999 - A method for designing primer sets for speciation studies in filamentous ascomycetes. Mycologia 91, 553-556.

Chowdappa P, Chethana CS, Pant RP, Bridge PD. 2014 - Multilocus gene phylogeny reveals occurrence of Colletotrichum cymbidiicola and C. cliviae on orchids in North East India. Journal of Plant Pathology 96, 327-334.

Damm U, Cannon PF, Woudenberg JHC, Crous PW. 2012 - The Colletotrichum acutatum complex. Studies in Mycology 73, 37-113.

Daykin ME, Miholland RD. 1984 - Ripe rot of muscadine grape caused by Colletotrichum gloeosporioides and its control. Phytopathology 74, 710-714.

Doyle VP, Oudemans PV, Rehner SA, Litt A. 2013 - Habitat and host indicate lineage identity in Colletotrichum gloeosporioides s.l. from wild and agricultural landscapes in North America. PLoS One 8: e62394.

Fang ZD.1998 - Plant disease research methods. China agriculture press, Beijing.

Fujian Provincial Bureau of Statistics. 2015 - FUJIAN RURAL STATISTICAL YEARBOOK-2015.

Hall TA.1999 - BioEdit: a user-friendly biological sequence alignment editor and analysis program for windows 95/98/NT. Nucleic Acids Symp Ser 41, 95-98.

Hillis DM, Bull JJ. 1993 - An empirical test of bootstrapping as a method for assessing confidence in phylogenetic analysis. Syst Biol 42, 182-192.

Hong SK, Wan GK, Yun HK, Choi KJ. 2008 - Morphological Variations, Genetic Diversity and Pathogenicity of Colletotrichum species Causing Grape Ripe Rot in Korea. Plant Pathology Journal 24, 269-278.

Huang F, Chen GQ, Hou X, Fu YS, Cai L, Hyde KD, Li HY. 2013 - Colletotrichum species associated with cultivated citrus in China. Fungal Diversity 61, 61-74.

Hyde KD, Cai L, Cannon PF, Crouch JA, Crous PW, Damm U, Goodwin PH, Chen H, Johnston PR, Jones EBG, Liu ZY, McKenzie EHC, Moriwaki J, Noireung P, Pennycook SR, Pfenning LH, Prihastuti H, Sato T, Shivas RG, Tan YP, Taylor PWJ, Weir BS, Yang YL, Zhang JZ. 2009 Colletotrichum - names in current use. Fungal Diversity 39, 147-182.

Katoh K, Toh H. 2008 - Recent developments in the MAFFT multiple sequence alignment program. Bioinformatics 9, 276-285.

Kishino H, Hasegawa M. 1989 - Evaluation of the maximum likelihood estimate of the evolutionary tree topologies from DNA sequence data. J Mol Evol 29, 170-179.

Lei Y, Liu XM, Chen T, Cai SH, Huang XZ. 2011 - Fujian grape industry situation and 
comparative advantage and development strategy. Fujian Fruits 2, 22-25.

Lu XL. 2005 - Studies on biochemistry and molecular biology of genetic diversity of grape cultivar and resistant appraisal of downy mildew. Sichuan Agricultural University, Sichuan.

Melksham KJ, Weckert MA, Steel CC. 2002 - An unusual bunch rot of grapes in sub-tropical regions of Australia caused by Colletotrichum acutatum. Australasian Plant Pathology 31, 193-194.

Moriwaki J, Tsukiboshi T. 2009 - Colletotrichum echinochloae, a new species on Japanese barnyard millet ( Echinochloa utilis ). Mycoscience 50, 273-280.

Nylander JAA. 2004 - MrModeltest V2. Program distributed by the author. Evolutionary Biology Centre, Uppsala University.

O’Donnell K, Cigelnik E. 1997 - Two divergent intra genomic rDNA ITS2 types within a monophyletic lineage of the fungus Fusarium are nonorthologous. Mol Phylogenet Evol 7, 103-116.

Peng LJ, Sun T, Yang YL, Cai L, Hyde KD, Bahkali AH, Liu ZY. 2013 - Colletotrichum species on grape in Guizhou and Yunnan provinces, China. Mycoscience 54, $29-41$.

Phoulivong S, Cai L, Chen H, Mckenzie EHC, Abdelsalam K, Chukeatirote E, Hyde KD. 2010 Colletotrichum gloeosporioides is not a common pathogen on tropical fruits. Fungal Diversity 44, 33-43.

Ronquist F, Huelsenbeck JP. 2003 - MrBayes 3:Bayesian phylogenetic inference under mixed models. Bioinformatics 19, 1572-1574.

Silva DN, Talhinhas P, Várzea V, Cai L, Paulo OS, Batista D. 2012 - Application of the Apn2/MAT locus to improve the systematics of the Colletotrichum gloeosporioides complex: an example from coffee (Coffea spp.) hosts. Mycologia 104, 396-409.

Southworth EA. 1891- Ripe Rot of Grapes and Apples. Journal of Mycology 6, 164-173.

Swofford DL. 2002 - PAUP*: phylogenetic analysis using parsimony (and other methods).Version 4.0b10. Sinauer Associates, Sunderland.

Tang DM.2015 - Situation of industry and recommendations for crystal grapes in Guizhou Province.Hebei Forestry Science and Technology 4,112-114.

Templeton MD, Rikkerink EHA, Solon SL, Crowhurst RN.1992-Cloning and molecular characterization of the glyceraldehyde-3-phosphate dehydrogenase encoding gene and cDNA from the plant pathogenic fungus Glomerella cingulata. Gene 122, 225-230.

Thompson JD, Gibson TJ, Plewniak F, Jeanmougin F, Higgins DG. 1997 - The Clustsal X windows interface:Flexible strategies for multiple sequence alignment aided by quality analysis tools. Nucleic Acids Res 24, 4876-4882.

Weir BS, Johnston PR, 2010 - Characterisation and neotypification of Gloeosporium kaki Hori as Colletotrichum horii nom. nov. Mycotaxon 111, 209-219.

Weir BS, Johnston PR, Damm U. 2012 - The Colletotrichum gloeosporioides species complex. Studies in Mycology 73, 115-180.

White TJ, Bruns T, Lee S, Taylor J. 1990 - Amplification and direct sequencing of fungal ribosomal RNA genes for phylogenetics. PCR Protocols 38, 315-322.

Whitelaw-Weckert MA, Curtin SJ, Huang R, Steel CC, Blanchard CL, Roffey PE. 2007 Phylogenetic relationships and pathogenicity of Colletotrichum acutatum isolates from grape in subtropical Australia. Plant Pathology 56, 448-463.

Wikee S, Cai L, Pairin N, Mckenzie EHC, Su YY, Chukeatirote E, Thi HN, Bahkali AH, Moslem MA, Abdelsalam K, Hyde KD. 2011 - Colletotrichum species from Jasmine (Jasminum sambac). Fungal Diversity 46, 171-182.

Yamamoto J, Sato T, Tomioka K. 1999 - Occurrence of ripe rot of grapes (Vitis vinifera L.) caused by Colletotrichum acutatum Simmonds ex Simmonds. Japanese Journal of Phytopathology 65, 83-86.

Yan JY, Jayawardena MMRS, Goonasekara ID, Wang Y, Zhang W, Liu M, Huang JB, Wang ZY, 
Shang JJ, Peng YL, Bahkali A, Hyde KD, Li XH. 2015 - Diverse species of Colletotrichum associated with grapevine anthracnose in China. Fungal Diversity 71, 233-246. 